\title{
Use of Ultrasound for Diagnosis of Clavicle Fractures in the Emergency Department
}

\author{
Sinan Karacabey ${ }^{1, *}$, Erkman Sanrı ${ }^{1}$
}

${ }^{1}$ Emergency Medicine Department, Marmara University School of Medicine Pendik Training and Research Hospital, Istanbul, Turkey

\section{*Correspondence}

karacabeysinan@gmail.com

(Sinan Karacabey)

\begin{abstract}
Background: Plain radiography is the method for diagnosing the fracture of the clavicle. The use of US to diagnose clavicle fracture has several advantages when compared to radiography. It could prevent the patient from radiation exposure, especially in vulnerable populations. It may also expedite the diagnosis and decrease the length of stay in the ED. In this study we aimed to discuss the diagnostic success of ultrasonography versus x-ray. Materials and Method: All patients admitted to the emergency department with a shoulder trauma were investigated for their eligibility to be included in the study. A standardized ultrasonography performed by the same investigator to vizualise clavicle from sternal junction through acromial junction. After ultrasonography, plain radiography was performed. Results: The mean age was 45.53 $(\min =18 ; \max =86 ; \mathrm{SD}=18.791)$ years and $72.7 \%$ were male. Among all patients, 42 clavicula fractures were detected via graphy and $26(62 \%)$ were seen in males and 57 patients with no clavicle fractures. The sensitivity of US to radiographically detected fracture was $92.86 \%(95 \% \mathrm{CI}, 80.52 \%$ to $98.5 \%)$, and the specificity was $98.25 \%(95 \%$ $\mathrm{CI}=90.61 \%$ to $99.96 \%)$. The PPV was $97.5 \%(84.8 \%$ to $99.63 \%)$ and the NPV was $94.92 \%(95 \% \mathrm{CI}=86.23 \%$ to $98.23 \%)$. Conclusions: Ultrasonography is a good alternative for diagnosing clavicle fracture. Future studies should examine the use of ultrasonography as a method for diagnosing of clavicle fracture by emergency physicians with only basic ultrasonographic training.
\end{abstract}

\section{Keywords}

Trauma, Clavicle fracture, Ultrasonography

\section{Introduction}

Musculoskeletal injuries are common and represent a large number of patients presenting to the Emergency Departments (EDs) [1]. Clavicle fractures constitute $2-4 \%$ of all fractures [2]. The clavicle has essential functions, primarily to stabilize the shoulder and allow full range of movement of the arm. Additionally, muscles attached to the clavicle contribute to a person's physical appearance, protects vital neurovascular structures and support respiratory function. Clavicle fractures lead to a loss of these essential functions [3]. Management of clavicle fracture is straightforward, but it requires imaging for confirmation of diagnosis [4].

Radiography is traditionally used to diagnose bone fractures, but this can be time-consuming, increase waiting times and stay in the ED and result in radiation exposure. General use of ultrasonography (US) has increased in the ED in the last two decades. US has a wide range of use in $\mathrm{ED}$ (abdominal, cardiac emergencies, trauma, etc.) [5, 6]. Fractures exceptionally long bone fractures are well diagnosed by US [7, 8]. There were studies about diagnosing clavicle fractures in the pediatric population with US, and these studies showed US could accurately diagnose clavicle fractures $[9,10]$.

The use of US to diagnose clavicle fracture has several advantages compared to radiography. It could eliminate radiation exposure, expedite the diagnosis and decrease the length of stay in the ED.

Prior studies showed that US has high sensitivity and specificity in long bone fractures [11-13]. However, previous studies about use of US for the diagnosis of clavicle fractures have focused on specific patient populations such as children and have not addressed the general adult population $[9,14]$.

In this study, we aimed to discuss US diagnostic accuracy in adult patients with clavicle fractures compared to radiography.

\section{Materials and method}

This was a prospective study comparing the diagnostic accuracy of US versus radiography Written informed consent was obtained from all patients. The study was enrolled between January 2016-September 2016.

All patients seen with shoulder trauma in the ED, who met inclusion and exclusion criteria, were included in the study. All non-pregnant patients over 18-year-old with written informed 


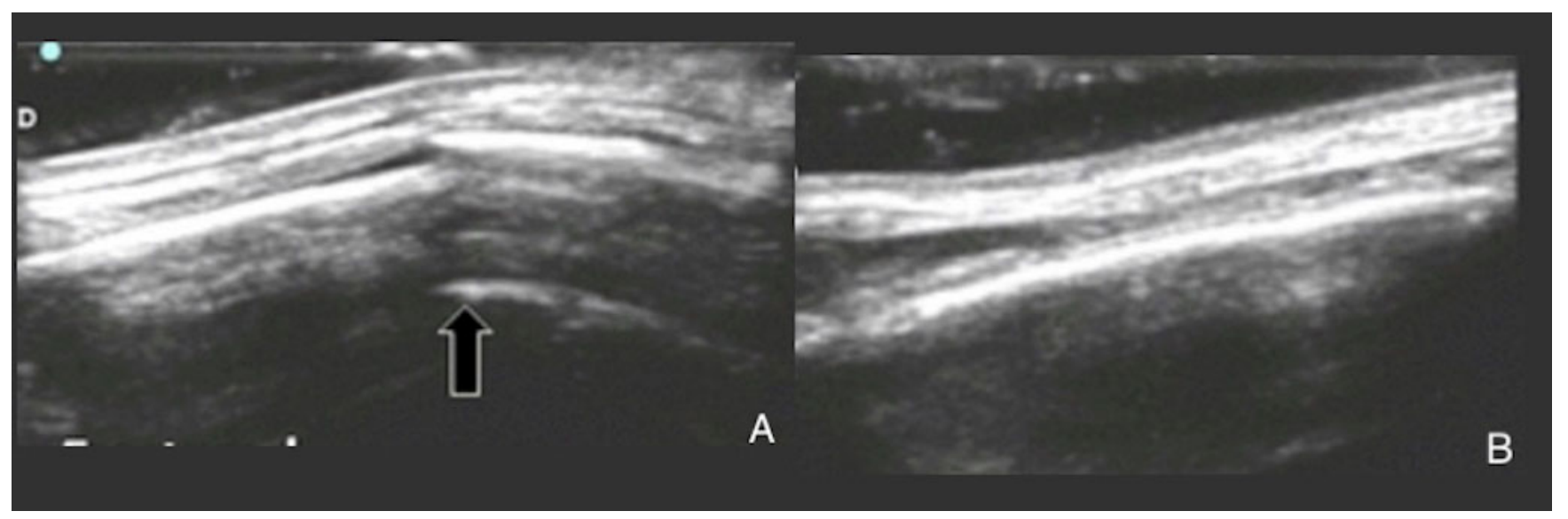

F I G U R E 1. Ultrasound images of fractured (A) and normal (B) clavicle.

Arrow point to region of cortical interruption on US.

consent were included in the study. Patients with altered mental status, multisystem trauma, intubated, hemodynamic unstable, with open shoulder wounds, or with a diagnosis before presenting to the ED were excluded from the study.

All patients were given standard analgesic treatment per protocol for trauma patients. After signing informed consent, the study investigator performed a physical examination, and patients suspected of clavicle fracture were enrolled.

All US scans were performed by the same experienced investigator. The investigator completed an US course on musculoskeletal ultrasonography and had 5-year experience with bedside US. A US device with a $7.5 \mathrm{MHz}$ linear probe were used for the US. The clavicle was visualized from the sternal junction to the acromial junction. Clavicle fracture diagnosis was based on seeing cortical bone disruption on bone motion with the respiratory cycle. These US findings of clavicle fractures are consistent with prior studies $[15,16]$. An Emergency Physicians performed all the bedside US's. All outcome measures of the study (including interpretation of US images) were also interpreted by EMP's.

After completion of the US, patients proceeded to plain radiography consistent with the protocol for clavicle fractures (Fig. 2). The results of the plain radiography were analyzed by a separate physician who was blinded to the US results and recorded those patients with a clavicle fracture. The blinded physician also had five-year experience reading radiography and specialized in musculoskeletal radiography.

All data were collected and recorded in SPSS version 23 (SPSS Inc., Chicago, IL). Statistical analysis was done by a clinician blinded to the patients. Data were used to calculate sensitivity, specificity, and positive and negative likelihood ratios with $95 \%$ confidential intervals and positive and negative predictive values. A sample size of 96 was able to detect a $10 \%$ difference in AUCs with a significance level of 0.05 and $80 \%$ power. A final sample size of 99 patients was considered adequate to compensate of inconclusive tests.

\section{Results}

Ninety-nine patients were enrolled from January/2016 September/2016. Over the study period, 145 patients were

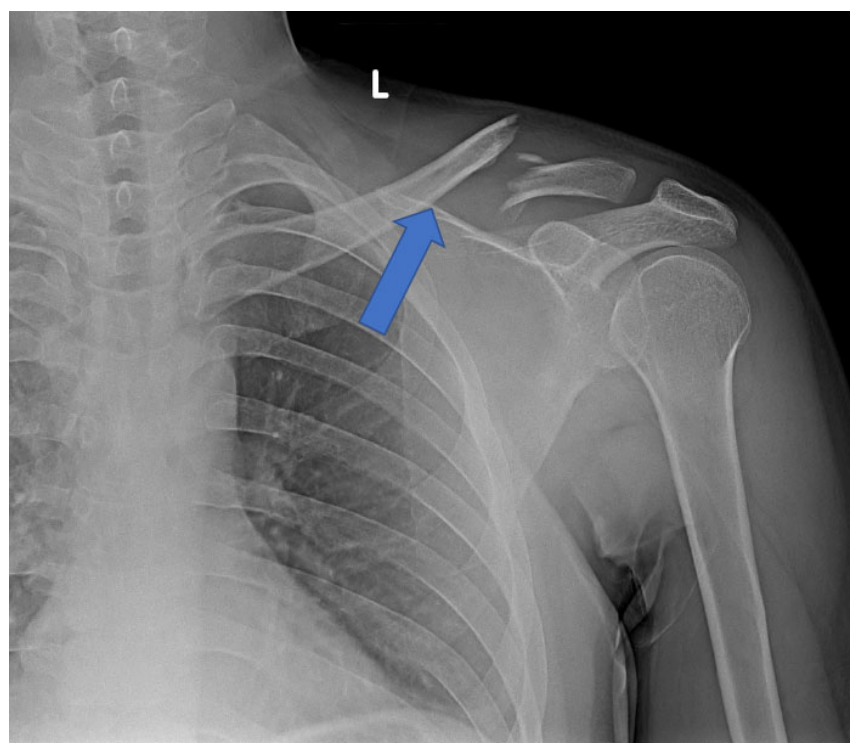

F IGURE 2. Left mid-clavicular fracture on plain radiography.

evaluated in the ED for shoulder trauma, 35 did not meet eligibility criteria, 110 were enrolled in the study, and 11 were excluded. The final study population was 99 patients.

The mean age was $45.53(\min =18 ; \max =86 ; \mathrm{SD}=18.791)$ years and $72.7 \%$ were male. Among all patients, 42 clavicula fractures were detected via radiography, and $26(62 \%)$ were seen in males. Fifty-seven patients had no clavicle fractures. Patients' place of fracture and treatment methods of these patients summarized in Table 1.

Table 2 shows US images for patients who had no clavicle fractures on plain radiography. Three false-negative cases were recorded: these were fractures seen on plain radiographs that were not seen in the US. All these three false-negative cases were nonangulated non-displaced greenstick fractures, and the orthopedic surgeons recommended no treatment.

There were 12 nonangulated fractures successfully diagnosed by US and one false-positive. The false positive was likely due to superficial soft tissue artifacts. The patient was a heavy-weight lifter with a previous clavicle fracture. In plain radiography, callus tissue was seen in the trauma area, 
TA B L E 1. Place of fracture and treatment preferences. Place of Fracture Treatment Preferences

\begin{tabular}{lccc} 
& Surgical & Nonsurgical \\
Proximal & 2 & 10 & 12 \\
Mid & 6 & 9 & 15 \\
Distal & 3 & 12 & 15 \\
Total & 11 & 31 & 42 \\
\hline
\end{tabular}

which was diagnosed as a past fracture. The US's sensitivity to radiographically detected fracture was $92.86 \%$ (95\% CI, $80.52 \%$ to $98.5 \%)$, and the specificity was $98.25 \%(95 \% \mathrm{CI}=$ $90.61 \%$ to $99.96 \%)$. The PPV was $97.5 \%(84.8 \%$ to $99.63 \%)$ and the NPV was $94.92 \%$ (95\% CI $=86.23 \%$ to $98.23 \%)$; + LR, 52.93 (7.57 - 369.99); -LR, 0.07 (0.02 - 0.21).

\section{Discussion}

Bedside US has wide use in the ED, especially in skeletal fractures in adults. This study is the first to discuss the use of US in the diagnosis of clavicle fractures. In this study, we report that the US can accurately diagnose clavicle fractures in adult patients compared to radiography. In the pediatric literature, two studies in infants comprising 41 and 49 patients showed the US's use in the diagnosis of clavicle fracture was comparable to radiographs. In both studies, the US was performed by radiologists, not ED physicians [17, 18].

In another study conducted in 103 children in the ED, US compared to radiographs, showed $95 \%$ sensitivity, 96\% specificity, 95\% PPV, and 96\% NPV in diagnosing clavicle fracture. They reported two false positives and two false-negatives of missed hairline fractures. The limitation of this study was similar to our study. They have one physician who performed all the US scans that may limit the generalization of their results [9].

For medico-legal reasons, many physicians in the ED choose imaging methods to confirm clavicle fractures rather than relying purely on clinical judgment. The US shows a good alternative to radiography to satisfy these medico-legal concerns. In other studies, the US's use in ED has shown to shorten the length of stay and lower costs. Also, the use of US avoids radiation exposure. We believe that the US should be the first diagnostic method to limit time and costs as well as radiation exposure.

Overall our results suggest that with a specificity $98.3 \%$ if the US is positive for a clavicle fracture and the patient has no deficits or gross deformity, a confirmatory radiograph is unnecessary. False-positive results lead to supportive care for the patients. Also, if the US is negative for fracture, there is no need for radiography because these are only non-displaced fractures that are misdiagnosed by the US. Treatment in either case is unchanged with a sling for 2 - 3 weeks.

Our study showed that formally trained and experienced EMP's could accurately diagnose clavicle fractures with high accuracy at the bedside. However, we cannot generalize our results because a single individual performed all the US scans.

The use of US has several advantages for diagnosing bone fractures and also clavicle fractures. First of all, avoiding
TA B L E 2. Results for US Versus Radiography. Plain Radiography+ Plain Radiography -

\begin{tabular}{lcc} 
Ultrasound+ & 39 & 1 \\
Ultrasound- & 3 & 56 \\
\hline
\end{tabular}

Sensitivity, 92.86\% (95\% CI, 80.52\% to 98.5\%); Specificity, 98.25\% (CI, 90.61\%-99.96\%);

PPV, 97.5\% (84.8\% to 99.63\%); NPV, 94.92\% (CI, $86.23 \%$ to 98.23\%); + LR, 52.93 (7.57-369.99); -LR, 0.07 (0.02 - 0.21).

excessive radiation exposure in specific populations such as pregnant patients. Also, US use may ease taking images when it is difficult to position or transport the patient. Bedside US avoids the patient transport to the radiology unit and transfer the patients from the bed to the table and also avoids moving the injured body part. Rapidly diagnosing these patients will also relieve EDs from overcapacity.

Our study has several limitations; the main limitation in our study was that the gold standard of computerized tomography was not utilized. However, radiography is as accurate as computerized tomography for diagnosing clavicle fracture. For this reason, this was not an important limitation. This study was conducted at a single site by a small number of investigators, all of whom had prior experience with US.

This study was conducted at a single site by a small number of investigators, all of whom had prior experience with the US. For this reason, we can not generalize our results. Physicians were not blinded to the clinical information of the patients; this may cause an operator bias. The costs and speed of both imaging techniques were not compared. However, in our opinion, the US was faster and cheaper than radiography in most instances. All the data were recorded as fractures or no fracture. The place and the type of fracture were not noted.

In conclusion, this study shows that emergency physicians can accurately diagnose clavicle fractures in ED with bedside US. These findings may challenge the need for radiography for diagnosing clavicle fractures.

\section{ACKNOWLEDGMENTS}

Thanks to all the peer reviewers and editors for their opinions and suggestions.

\section{CONFLICT OF INTEREST}

We declare that we do not have any commercial or associative interest that represents a conflict of interest in connection with the work submitted.

\section{ETHICAL APPROVAL}

The study was approved by Bozok University institutional review board (22.02.2016/25/06/01).

\section{REFERENCES}

[1] Control CfD, Prevention. National Hospital Ambulatory Medical Care Survey: 2010 Emergency Department Summary Tables. Centers for 
Disease Control and Prevention. 2016.

[2] Herteleer M, Winckelmans T, Hoekstra H, et al. Epidemiology of clavicle fractures in a level 1 trauma center in Belgium. Eur J Trauma Emerg Surg. 2018;44:717-726

[3] Wiesel B, Nagda S, Mehta S, et al. Management of midshaft clavicle fractures in adults. J Am Acad Orthop Surg. 2018;26:e468-e476.

[4] Anderson K. Evaluation and treatment of distal clavicle fractures. Clin Sports Med. 2003;22:319-326, vii.

[5] Dogan S, Kalafat UM, Yüksel B, et al. Use of radiography and ultrasonography for nasal fracture identification in children under 18 years of age presenting to the ED. Am J Emerg Med. 2017;35:465-468.

[6] Park YH, Jung RB, Lee YG, et al. Does the use of bedside ultrasonography reduce emergency department length of stay for patients with renal colic?: a pilot study. Clin Exp Emerg Med. 2016;3:197-203.

[7] McNeil CR, McManus J, Mehta S. The accuracy of portable ultrasonography to diagnose fractures in an austere environment. Prehosp Emerg Care. 2009; 13:50-52.

[8] Marshburn TH, Legome E, Sargsyan A, et al. Goal-directed ultrasound in the detection of long-bone fractures. J Trauma. 2004;57:329-332.

[9] Cross KP, Warkentine FH, Kim IK, et al. Bedside ultrasound diagnosis of clavicle fractures in the pediatric emergency department. Acad Emerg Med. 2010;17:687-693.

[10] Chien M, Bulloch B, Garcia-Filion P, et al. Bedside ultrasound in the diagnosis of pediatric clavicle fractures. Pediatr Emerg Care. 2011;27:1038-1041.

[11] Patten RM, Mack LA, Wang KY, et al. Non-displaced fractures of the greater tuberosity of the humerus: sonographic detection. Radiology. 1992;182:201-204.

[12] Weinberg ER, Tunik MG, Tsung JW. Accuracy of clinician-performed point-of-care ultrasound for the diagnosis of fractures in children and young adults. Injury. 2010;41:862-868.

[13] Chen L, Kim Y, Moore CL. Diagnosis and guided reduction of forearm fractures in children using bedside ultrasound. Pediatr Emerg Care. 2007;23:528-531.

[14] Moritz J, Berthold L, Soenksen S. Ultrasound in diagnosis of fractures in children: unnecessary harassment or useful addition to X-ray? Ultraschall Med. 2008;29:267-274.

[15] Ekpioðlu F, Altýnok D, Uslu MM. Ultrasonographic findings in pediatric fractures. Turk J Pediatr. 2003;45:136-140.

[16] Sferopoulos N. Fracture separation of the medial clavicular epiphysis: ultrasonography findings. Arch Orthop Trauma Surg. 2003;123:367-369.

[17] Katz R, Landman J, Dulitzky F, et al. Fracture of the clavicle in the newborn. An ultrasound diagnosis. J Ultrasound Med. 1988;7:21-23.

[18] Blab E, Geissler W, Rokitansky A. Sonographic management of infantile clavicular fractures. Pediatr Surg Int. 1999;15:251-254.

How to cite this article:Sinan Karacabey, Erkman Sanr1. Use of Ultrasound for Diagnosis of Clavicle Fractures in the Emergency Department. Signa Vitae. 2021;17(1):139-142. doi:10.22514/sv.2020.16.0046. 